\title{
Magnetism and Conductivity Along Structural Domain Walls of $\mathrm{SrTiO}_{3}$
}

\author{
Yiftach Frenkel $^{1} \cdot$ Yanwu Xie ${ }^{2,3,4} \cdot$ Harold Y. Hwang $^{2,3} \cdot$ Beena Kalisky $^{1}$ \\ Received: 31 July 2019 / Accepted: 10 September 2019 / Published online: 16 November 2019 \\ (C) The Author(s) 2020, corrected publication 2020
}

\begin{abstract}
The interface between the oxide insulators $\mathrm{LaAlO}_{3}$ and $\mathrm{SrTiO}_{3}$ (LAO/STO) hosts a two-dimensional electron gas. The combination of interfacial conductivity and superconductivity at ultra-low temperatures with the physical phenomena of the oxide parent materials has fueled extensive research in the field since its discovery in 2004. Scanning superconducting quantum interference device (SQUID) measurements have shown that structural domain walls, formed below $105 \mathrm{~K}$, modulate the current flow at the interface and recently revealed weak magnetic signals along the same domain structure. Here we use scanning SQUID to investigate the temperature dependence of different electronic properties of the LAO/STO interface. We find correlation between magnetism and conductivity, which are both spatially modulated on the domain structure. This data suggests a possible relation between the populations of electrons participating in each order.
\end{abstract}

Keywords Oxide interfaces $\cdot$ Magnetism $\cdot$ Conductivity $\cdot$ Scanning SQUID microscopy $\cdot$ Polarity $\cdot$ Ferroelectricity

Strontium titanate (STO) is one of the most studied transition metal oxides in solid-state physics. It exhibits various unique properties such as very high dielectric constant at low temperatures [1], dilute superconductivity [2], and proximity to a quantum critical point [3] (between paraelectric and ferroelectric states). In addition, STO undergoes a structural phase transition at $105 \mathrm{~K}$ from cubic to tetragonal crystal structure [4]. In the tetragonal phase, the STO forms a dense network of structural domains separated by well-defined domain walls. Below $\sim 37 \mathrm{~K}$, the STO undergoes another transition where its dielectric constant rapidly increases, but a macroscopic ferroelectric state is not reached [5]. Salje et al. [6] used piezoelectric measurements

Beena Kalisky

beena@biu.ac.il

$1 \quad$ Physics Department, Institute of Nanotechnology and Advanced Materials, Bar Ilan University, 5290002 Ramat-Gan, Israel

2 Stanford Institute for Materials and Energy Sciences, SLAC National Accelerator Laboratory, Menlo Park, CA 94025, USA

3 Department of Applied Physics, Geballe Laboratory for Advanced Materials, Stanford University, 476 Lomita Mall, Stanford, CA 94305, USA

4 Department of Physics, Zhejiang University, Hangzhou 310027, China to suggest that instead of a global ferroelectric state, the STO structural domain walls develop polarity below $40 \mathrm{~K}$. Local measurements support this scenario [7]. Here we focus on properties that are modulated on the STO domain structure, and track their effects on the LAO/STO conductive interface [8-12]. Below $\sim 37 \mathrm{~K}$, the current flowing at the interface is modulated along the structural domain walls [13]. By combining transport with local imaging, it was shown that these modulations can induce significant anisotropy in the resistance of devices [14]. By using scanning stress measurements, where small changes in resistance are monitored as a function of local stress, indications of polarity at domain walls were found and were related to the spatial modulation in the current flow [7].

Here we use a scanning SQUID microscope to investigate the temperature dependence of different electronic properties of the LAO/STO interface [13] and in particular their modulations along the STO domain walls. The static magnetic landscape (Fig. 1 left column) corresponds to flux originating in local magnetic moments. This is a map of the magnetic field that threads the SQUID's pick-up loop (see illustrations in Fig. 1). The spatial distribution of the current flow (middle column) is obtained by mapping the Oersted magnetic fields that are generated by $\mathrm{AC}$ current flowing in the sample. The right column shows scanning stress measurements, which are maps of small changes in the four- 
Fig. 1 The temperature dependence of modulated magnetic and electronic properties along STO domain walls. Static magnetic signal (left), spatial distribution of current (middle), and electrical response to stress (right) simultaneously imaged by scanning SQUID. Both the response to stress and the current modulations increase as the temperature is lowered. The modulations of the magnetic signal show the opposite behavior, a reduced modulation at lower temperatures
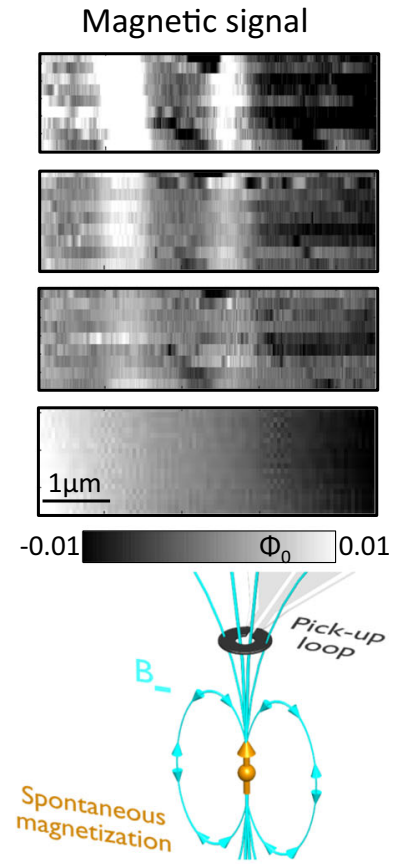

\section{(1)}
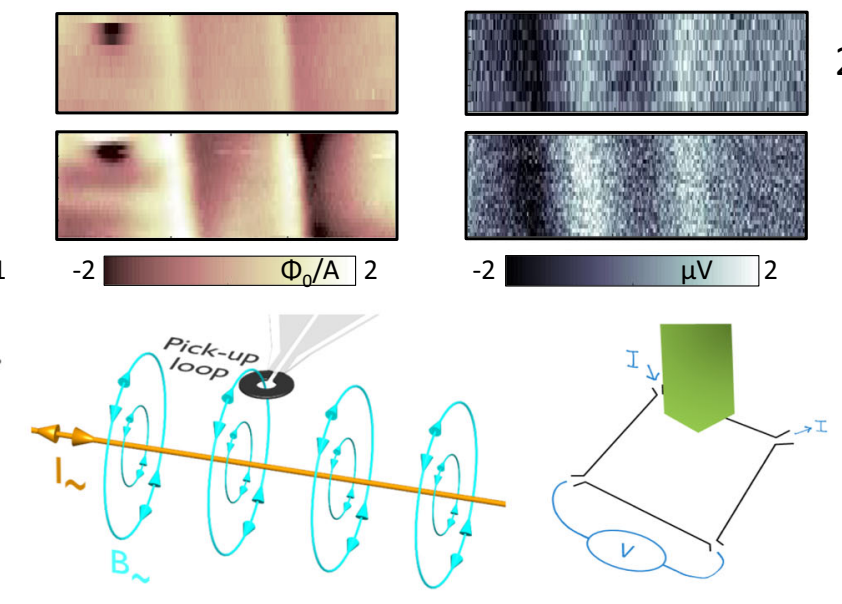

$5 K$
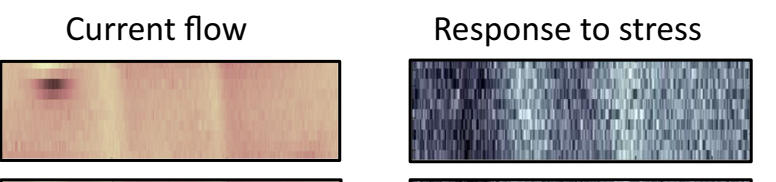

$30 \mathrm{~K}$

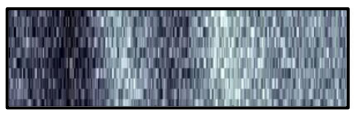

$25 K$

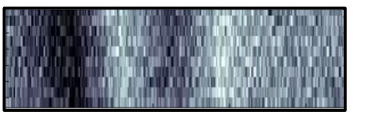

$20 K$

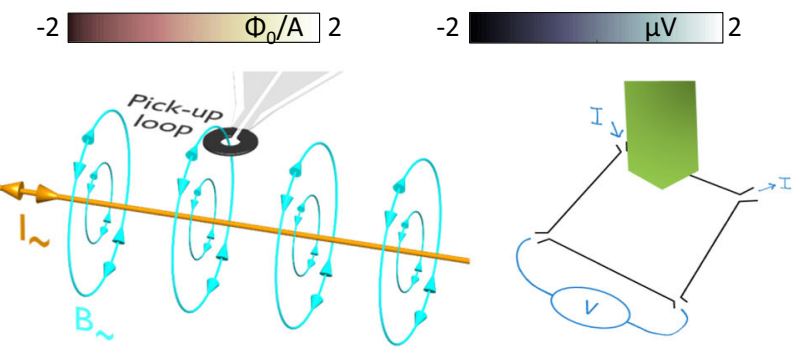

Open Access This article is licensed under a Creative Commons Attribution 4.0 International License, which permits use, sharing, adaptation, distribution and reproduction in any medium or format, as long as you give appropriate credit to the original author(s) and the source, provide a link to the Creative Commons licence, and indicate if changes were made. The images or other third party material in this article are included in the article's Creative Commons licence, unless indicated otherwise in a credit line to the material. If material is not included in the article's Creative Commons licence and your intended use is not permitted by statutory regulation or exceeds the permitted use, you will need to obtain permission directly from the copyright holder. To view a copy of this licence, visit http://creativecommons.org/licenses/by/4.0/.

\section{References}

1. Sakudo, T., Unoki, H.: Dielectric properties of $\mathrm{SrTiO} 3$ at low temperatures. Phys. Reivew Lett. 26, 851-853 (1971). https://doi.org/ 10.1103/PhysRevLett.26.851

2. Koonce, C.S., Cohen, M.L., Schooley, J.F., Hosler, W.R., Pfeiffer, E.R.: Superconducting transition temperatures of semiconducting SrTiO3. Phys. Rev. 163, 380-390 (1967). https://doi.org/10.1103/ PhysRev.163.380

3. Edge, J.M., Kedem, Y., Aschauer, U., Spaldin, N.A., Balatsky, A.V.: Quantum critical origin of the superconducting dome in SrTiO3. Phys. Rev. Lett. 115, 1-5 (2015). https://doi.org/10.1103/ PhysRevLett.115.247002

4. Cowley, R.A.: Lattice dynamics and phase transitions of strontium titanate. Phys. Rev. 134, A981-A997 (1964)

5. Rowley, S.E., Spalek, L.J., Smith, R.P., Dean, M.P.M., Itoh, M., Scott, J.F., Lonzarich, G.G., Saxena, S.S.: Ferroelectric quantum criticality. Nat. Phys. 10, 367-372 (2014). https://doi.org/10.1038/ nphys 2924

6. Salje, E.K.H., Aktas, O., Carpenter, M.A., Laguta, V.V., Scott, J.F.: Domains within domains and walls within walls: evidence for polar domains in cryogenic SrTiO3. Phys. Rev. Lett. 111, 247603 (2013) 
7. Frenkel, Y., Haham, N., Shperber, Y., Bell, C., Xie, Y., Chen, Z., Hikita, Y., Hwang, H.Y., Salje, E.K.H., Kalisky, B.: Imaging and tuning polarity at SrTiO3 domain walls. Nat. Mater. 16, 1203 (2017)

8. Ohtomo, A., Hwang, H.Y.: A high-mobility electron gas at the LaAlO3/SrTiO3 heterointerface. Nature. 427, 423-426 (2004). https://doi.org/10.1038/nature04773

9. Zubko, P., Gariglio, S., Gabay, M., Ghosez, P., Triscone, J.-M.: Interface physics in complex oxide heterostructures. Annu. Rev. Condens. Matter Phys. 2, 141-165 (2011)

10. Persky, E., Kalisky, B., Scanning, S.Q.U.I.D.: View of oxide interfaces. Adv. Mater. 30, 1-9 (2018). https://doi.org/10.1002/adma. 201706653

11. Wissberg, S., Kalisky, B.: Large-scale modulation in the superconducting properties of thin films due to domains in the SrTiO3 substrate. Phys. Rev. B. 95, 144510 (2017)

12. Noad, H., Spanton, E.M., Nowack, K.C., Inoue, H., Kim, M., Merz, T.A., Bell, C., Hikita, Y., Xu, R., Liu, W., Vailionis, A., Hwang, H.Y., Moler, K.A.: Variation in superconducting transition temperature due to tetragonal domains in two-dimensionally doped SrTiO3. Phys. Rev. B - Condens. Matter Mater. Phys. 94, 1-12 (2016). https://doi.org/10.1103/PhysRevB.94.174516
13. Kalisky, B., Spanton, E.M., Noad, H., Kirtley, J.R., Nowack, K.C., Bell, C., Sato, H.K., Hosoda, M., Xie, Y., Hikita, Y., Woltmann, C., Pfanzelt, G., Jany, R., Richter, C., Hwang, H.Y., Mannhart, J., Moler, K.A.: Locally enhanced conductivity due to the tetragonal domain structure in LAO/STO heterointerfaces. Nat. Mater. 12, 1091 (2013)

14. Frenkel, Y., Haham, N., Shperber, Y., Bell, C., Xie, Y., Chen, Z., Hikita, Y., Hwang, H.Y., Kalisky, B.: Anisotropic transport at the LaAlO 3 /SrTiO 3 interface explained by microscopic imaging of channel-flow over SrTiO 3 domains. ACS Appl. Mater. Interfaces. 8, 12514-12519 (2016)

15. Christensen, D.V., Frenkel, Y., Chen, Y.Z., Xie, Y.W., Chen, Z.Y., Hikita, Y., Smith, A., Klein, L., Hwang, H.Y., Pryds, N., Kalisky, B.: Strain-tunable magnetism at oxide domain walls. Nat. Phys. 15, 269-274 (2019). https://doi.org/10.1038/s41567-018-0363-x

16. Y.-Y. Pai, A. Tylan-Tyler, P. Irvin, J. Levy, LaAlO3/SrTiO3: a Tale of Two Magnetisms, (2016) 1-46

Publisher's Note Springer Nature remains neutral with regard to jurisdictional claims in published maps and institutional affiliations. 